\title{
COBORDISM OPERATIONS AND SINGULARITIES OF MAPS
}

\author{
BY CLINT MCCRORY 1
}

Communicated by Glen Bredon, October 30, 1975

If $f$ is a differentiable map of smooth manifolds, the critical set $\Sigma(f)$ is not a manifold, in general. However, there is a canonical resolution of the singularities of $\Sigma(f)$ (for generic $f$ ), due to I. Porteous [6]. This resolution can be used to give a geometric description of T. tom Dieck's Steenrod operations in unoriented cobordism [7]. This was suggested to me by Jack Morava, as a parallel to my discription of ordinary mod 2 Steenrod operations using branching cycles of maps of $n$-circuits [5].

1. Singularities of vector bundle maps. Let $\xi^{n}=(E \rightarrow X)$ and $\eta^{p}=(F$ $\rightarrow X$ ) be real vector bundles over the smooth manifold $X$ (without boundary), and let $g: E \rightarrow F$ be a vector bundle map. That is, $g$ is smooth, and for each $x \in X, g$ sends the fiber $E_{x}$ to the fiber $F_{x}$ by a linear map $g_{x}$. The critical set $\Sigma(g)$ is $\left\{x \in X, \operatorname{rank}\left(g_{x}\right)<\min (n, p)\right\}$. Let $P(\xi)=(P(E) \rightarrow X)$ be the projectification of $\xi$, i.e. the bundle whose fiber over $x$ is the set of one-dimensional subspaces of $E_{x}$. Set $\widetilde{\Sigma}(g)=\{l \in P(E), l \subset$ kernel $(g)\}$. The projection $\widetilde{\Sigma}(g) \rightarrow X$ is proper, and if $n \leqslant p$, its image is $\Sigma(g)$. (If $n>p$, its image is all of $X$.)

LEMmA. (a) If $g: \xi^{n} \rightarrow \eta^{p}$ is a generic vector bundle map [4] over the d-manifold $X, \widetilde{\Sigma}(g)$ is $a(d-i)$-manifold, where $i=p-n+1$.

(b) If $h: \xi^{n} \rightarrow \eta^{p}$ is another such map, $\widetilde{\Sigma}(h) \rightarrow X$ is properly cobordant with $\widetilde{\Sigma}(g) \rightarrow X$.

This lemma is proved by considering the canonical bundle map $G$ over $\operatorname{Hom}(\xi, \eta)$. A vector bundle map $g: \xi \rightarrow \eta$ defines a section of $\operatorname{Hom}(\xi, \eta) \rightarrow$ $X$, and $\widetilde{\Sigma}(g) \rightarrow X$ is the pull-back of $\widetilde{\Sigma}(G) \rightarrow \operatorname{Hom}(\xi, \eta)$ by this section.

It follows from Quillen's geometric description of smooth unoriented cobordism theory $N^{*}$ [3] that this construction defines a natural transformation $\sigma: K(X) \rightarrow N^{*}(X)$. If $K(X)$ is defined as the set of all pairs $(\xi, \eta)$ of bundles over $X$, modulo the relation $(\xi \oplus \zeta, \eta \oplus \zeta) \sim(\xi, \eta), \sigma$ is induced by $(\xi, \eta) \longmapsto$ $\widetilde{\Sigma}(g)$, where $g: \xi \rightarrow \eta$ is a generic map. A dual $\bar{\sigma}$ is defined by $\bar{\sigma}[\xi, \eta]=$ $\sigma[\eta, \xi]$.

AMS (MOS) subject classifications (1970). Primary 55G25, 57D45; Secondary 57D20, 57D75.

Key words and phrases. Critical set, singularity, cobordism, Steenrod operations, vector bundle map.

${ }^{1}$ Support in part by NSF grant GP-43128. 
$\sigma$ determines a family of stable cobordism characteristic classes $\sigma_{i}, i \in \mathbf{Z}$, by setting $\sigma_{i}\left(\eta^{p}\right)=\sigma\left(\epsilon^{n}, \eta^{p}\right) \in N^{i}(X)$, where $\epsilon^{n}$ is the trivial $n$-bundle over $X$, $n=p-i+1$. If $\bar{\xi}$ is a stable inverse for $\xi, \sigma_{i}(\bar{\xi})=\bar{\sigma}_{i}(\xi)$.

2. Steenrod operations. Thom's definition of characteristic classes gives a bijection between stable operations on $N^{*}$ and stable $N^{*}$ characteristic classes. Let $\theta^{i}$ be the operation corresponding to the characteristic class $\sigma_{i}$.

Our main result is the following relation between $\theta=\Sigma_{i} \theta^{i}$ and tom Dieck's internal Steenrod operation $R[7, \mathrm{p} .394]$. Let $P^{-i}$ be the cobordism operation of degree $-i$ which sends $Z \rightarrow X$ to the composition $\left(\mathbf{R} P^{i} \times Z\right) \rightarrow Z \rightarrow X$, where $\mathbf{R} P^{i}$ is real projective $i$-space.

THEOREM $\theta .=P R$.

In other words, if $\alpha \in N^{q}(X), \theta^{i}(\alpha)=\Sigma_{j} P^{i-j} R^{j}(\alpha)$. Since $P^{i-j}=0$ for $j<$ $i$ and $R^{j}(\alpha)=0$ for $j>q$, this sum is finite.

It follows that $\theta$ corresponds to the "expanded square" operation in unoriented piecewise-linear cobordism [1].

This theorem is a consequence of the observation that $\bar{\sigma}_{i}\left(\xi^{n}\right)=\pi_{*}\left(e^{n+i-1}\right)$ for $i>-n$, where $\pi: P(E) \rightarrow X$ is the projection and $e$ is the cobordism Euler class of the (dual) canonical line bundle on $P(E)$. (For $i \leqslant-n, \bar{\sigma}_{i}(\xi)$ is represented by $P\left(\xi \oplus \epsilon^{k}\right), k=-n-i+1$.)

REMARK. Conner and Floyd's cobordism Stiefel-Whitney classes $w_{i}(\xi)$ (cf. [3]) are defined by the relation $\Sigma_{i}\left(\pi^{*} w_{i}\right) e^{n-i}=0$. Thus $\Sigma_{i} w_{i} \bar{\sigma}_{k-i}=0$ for $k$ $>0$.

3. Bordism operations (cf. [5]). There are dual actions of both $\theta$ and $R$ on smooth unoriented bordism theory $N_{*}$. If $M$ is a closed $n$-manifold, and $[M] \in$ $N_{n}(M)$ is the class of the identity map, $\theta^{i}[M]$ is represented by $\widetilde{\Sigma}(d f)$, where $f: M^{n} \rightarrow R^{n+i-1}$ is a generic smooth map. The following result is analogous to Thom's nonembedding theorem using ordinary Steenrod operations.

COROLlARY 1. If the locally triangulable space $X$ immerses topologically in $\mathbf{R}^{n}$, then $R^{i}$ is zero on $N_{j}(X)$ for $i+j>n$.

The action of $R^{i}$ on the bordism of a point is given by the "quadratic construction"

$$
Q_{k}(M)=M \times M \times S^{k-1} /(x, y, s) \sim(y, x,-s), \quad k=-n-i+1 .
$$

COROLlARY 2. If $M$ is a closed manifold, $Q_{k}(M)$ is cobordant with $P\left(T M \oplus \epsilon^{k}\right), k \geqslant 1$.

In fact, $M \times M \times D^{k} /(x, y, s) \sim(y, x,-s)$ minus an open tubular neighborhood of $\{[x, x, 0]\}$ is a cobordism between them. This generalizes an argument of Conner and Floyd for $k=1$ [2, p. 62] . 
REMARK. "Steenrod" operations in complex cobordism can be defined in the same way as $\theta^{i}$, by using complex vector bundles. Furthermore, replacing lines in $\xi$ by $k$-planes in $\xi$ yields a family of geometric operations $\theta_{(k)}^{i}$ for each $k$.

\section{REFERENCES}

1. S. Buoncristiano, C. P. Rourke and B. J. Sanderson, A geometric approach to homology theory, London Math. Soc. Lecture Notes in Math. (to appear).

2. P. E. Conner and E. E. Floyd, Differentiable periodic maps, Ergebnisse der Mathematik und ihrer Grenzgebiete, N. F., Band 33, Springer-Verlag, Berlin, 1964. MR 31 \#750.

3. M. Karoubi, Corbordisme et groupes formels (d' après D. Quillen et T. tom Dieck), Séminaire Bourbaki 1971/72, no. 408, Lecture Notes in Math., vol. 317, Springer-Verlag, Berlin and New York, 1973.

4. R. MacPherson, Generic vector bundle maps, Dynamical Systems (Proc. Sympos., Univ. of Bahia, Salvador, 1971), Academic Press, New York, 1973, pp. 165-175. MR 49 \#3962.

5. C. McCrory, Geometric homology operations, Advances in Math. (to appear).

6. I. R. Porteous, Simple singularities of maps, Proc. Liverpool Singularities-Sympos., I (1969/70), Lecture Notes in Math., vol. 192, Springer-Verlag, Berlin and New York, 1971, pp. 286-307. MR 45 \#2723.

7. T. tom Dieck, Steenrod-Operationen in Kobordismen-Theorien, Math. Z. 107 (1968), 380-401. MR 39 \#6302.

DEPARTMENT OF MATHEMATICS, BROWN UNIVERSITY, PROVIDENCE, RHODE ISLAND 02912 